\title{
Space-fractional Schrödinger equation for a quadrupolar triple Dirac- $\delta$ potential: Central Dirac- $\delta$ well and barrier cases
}

\author{
Jeffrey D. Tare* and Jose Perico H. Esguerra \\ National Institute of Physics, University of the Philippines-Diliman, \\ Quezon City 1101, Philippines \\ *jeffreytare@gmail.com \\ www.nip.upd.edu.ph
}

Published 2 January 2015

\begin{abstract}
We solve the space-fractional Schrödinger equation for a quadrupolar triple Dirac- $\delta$ (QTD- $\delta$ ) potential for all energies using the momentum-space approach. For the $E<0$ solution, we consider two cases, i.e., when the strengths of the potential are $V_{0}>0$ (QTD- $\delta$ potential with central Dirac- $\delta$ well) and $V_{0}<0$ (QTD- $\delta$ potential with central Dirac- $\delta$ barrier) and derive expressions satisfied by the bound-state energy. For all fractional orders $\alpha$ considered, we find that there is one eigenenergy when $V_{0}>0$, and there are two eigenenergies when $V_{0}<0$. We also obtain both bound- and scattering-state $(E>0)$ wave functions and express them in terms of Fox's $H$-function.
\end{abstract}

Keywords: Fractional quantum mechanics; space-fractional Schrödinger equation; quadrupolar triple Dirac- $\delta$ potential; Fox's $H$-function.

\section{Introduction}

Applications of fractional quantum mechanics (FQM) developed by Laskin ${ }^{1,2}$ via constructing fractional path integral over paths of Lévy flights have gained interest over the past 13 years. The formulation offers generalization of some results obtained in the standard quantum mechanics (SQM). One of its interesting applications is to delta potentials. ${ }^{3}$ Here we present another application of FQM by considering a quadrupolar triple Dirac- $\delta(\mathrm{QTD}-\delta$ ) potential in one dimension, which was first analyzed by Patil ${ }^{4}$ in the framework of SQM.

The time-independent space-fractional Schrödinger equation (TISFSE) in the position representation reads ${ }^{2}$

$$
D_{\alpha}\left(-\hbar^{2} \Delta\right)^{\alpha / 2} \psi(x)+V(x) \psi(x)=E \psi(x), \quad 1<\alpha \leq 2,
$$

where $D_{\alpha}$ is the generalized quantum diffusion coefficient $\left[D_{2}=1 /(2 \mathrm{~m})\right.$ with $\mathrm{m}$ being the mass of the particle], $\psi(x)$ is the wave function, $V(x)$ is the potential, $E$

This is an Open Access article published by World Scientific Publishing Company. It is distributed under the terms of the Creative Commons Attribution 3.0 (CC-BY) License. Further distribution of this work is permitted, provided the original work is properly cited. 
is the energy, and $\left(-\hbar^{2} \Delta\right)^{\alpha / 2}$ is the quantum Riesz fractional derivative:

$$
\left(-\hbar^{2} \Delta\right)^{\alpha / 2} \psi(x)=\frac{1}{2 \pi \hbar} \int_{-\infty}^{\infty} d p e^{i p x / \hbar}|p|^{\alpha} \int_{-\infty}^{\infty} e^{-i p x^{\prime} / \hbar} \psi\left(x^{\prime}\right) d x^{\prime} .
$$

In the momentum representation the TISFSE can be expressed as $^{3}$

$$
D_{\alpha}|p|^{\alpha} \tilde{\psi}(p)+\frac{(\tilde{V} * \tilde{\psi})(p)}{2 \pi \hbar}=E \tilde{\psi}(p)
$$

where $\tilde{\psi}(p)=\int_{-\infty}^{\infty} e^{-i p x / \hbar} \psi(x) d x$ is the Fourier transform of $\psi(x)$, with the inverse transform $\psi(x)=(2 \pi \hbar)^{-1} \int_{-\infty}^{\infty} e^{i p x / \hbar} \tilde{\psi}(p) d p$, and $(\tilde{V} * \tilde{\psi})(p)$ is the convolution of $\tilde{V}(p)$ and $\tilde{\psi}(p):(\tilde{V} * \tilde{\psi})(p)=\int_{-\infty}^{\infty} \tilde{V}\left(p-p^{\prime}\right) \tilde{\psi}\left(p^{\prime}\right) d p^{\prime}$. In the next section we solve Eq. (1) for a QTD- $\delta$ potential using the momentum-space approach.

\section{QTD- $\delta$ Potential and Solutions to the TISFSE}

The interaction between an electron and a symmetric linear triatomic molecule can be modeled using the potential ${ }^{4} V(x)=V_{0}[\delta(x+a)-2 \delta(x)+\delta(x-a)]$, where $V_{0}$ is the strength and $a$ is the spacing between the atoms. Its Fourier transform and convolution with $\tilde{\psi}(p)$ are given, respectively, by $\tilde{V}(p)=V_{0}\left(e^{i a p / \hbar}-2+e^{-i a p / \hbar}\right)$ and $(\tilde{V} * \tilde{\psi})(p)=V_{0}\left[e^{i a p / \hbar} C_{0}(a)-2 C_{1}(0)+e^{-i a p / \hbar} C_{2}(a)\right]$, where the constants are defined as $C_{0}(a)=C_{2}(-a)=\int_{-\infty}^{\infty} e^{-i a p^{\prime} / \hbar} \tilde{\psi}\left(p^{\prime}\right) d p^{\prime}$ and $C_{1}(0)=\int_{-\infty}^{\infty} \tilde{\psi}\left(p^{\prime}\right) d p^{\prime}$. Substituting the expression for $(\tilde{V} * \tilde{\psi})(p)$ in Eq. (3), we obtain an expression for $\tilde{\psi}(p)$ :

$$
\tilde{\psi}(p)=\frac{-\gamma_{\alpha}}{\left(|p|^{\alpha}-E / D_{\alpha}\right)}\left[e^{i a p / \hbar} C_{0}(a)-2 C_{1}(0)+e^{-i a p / \hbar} C_{2}(a)\right],
$$

with $\gamma_{\alpha}=V_{0} / 2 \pi \hbar D_{\alpha}$. Below we solve the TISFSE for two separate cases, namely, $E<0$ and $E>0$.

Case I: $E<0$. For this case, let us first consider $V_{0}>0$ (central Dirac- $\delta$ well case) and define $\lambda^{\alpha}=-E / D_{\alpha}(\lambda>0)$. Equation (4) then becomes $\tilde{\psi}(p)=$ $-\gamma_{\alpha}\left(|p|^{\alpha}+\lambda^{\alpha}\right)^{-1}\left[e^{i a p / \hbar} C_{0}(a)-2 C_{1}(0)+e^{-i a p / \hbar} C_{2}(a)\right]$. When we use this expression to find $C_{0}, C_{1}, C_{2}$, an equation satisfied by bound-state energy can be derived, namely, $R \epsilon^{\alpha-1} A(\epsilon)=R^{3} \epsilon^{3(\alpha-1)}-B(\epsilon)$, where $\epsilon=a \lambda / \hbar, R=2 \pi \hbar^{\alpha} D_{\alpha} /\left(a^{\alpha-1} V_{0}\right)$, $A(\epsilon)=3 T^{2}(0)-4 T^{2}(\epsilon)+T^{2}(2 \epsilon), B(\epsilon)=2\left\{T(0)\left[T^{2}(0)-2 T^{2}(\epsilon)-T^{2}(2 \epsilon)\right]+\right.$ $\left.2 T^{2}(\epsilon) T(2 \epsilon)\right\}$, and $T(y)=2 \int_{0}^{\infty} \cos (y q)\left(q^{\alpha}+1\right)^{-1} d q(q=p / \lambda)$. Figure 1 shows plots of the functions $f(\alpha, \epsilon)=R \epsilon^{\alpha-1} A(\epsilon)$ and $h(\alpha, \epsilon)=R^{3} \epsilon^{3(\alpha-1)}-B(\epsilon)$ for $R=2$ and some values of $\alpha$. The corresponding eigenvalue for each $\alpha$ can be identified as the $\epsilon$-coordinate of the point of intersection of the two curves. For the central Dirac- $\delta$ barrier case, $V_{0}<0$ [letting $V_{0}=-g(g>0)$ ], the following energy equations can be derived: (i) $T(2 \epsilon)=T(0)-Q \epsilon^{\alpha-1}$ and (ii) $T(0)-T(2 \epsilon)+2 Q \epsilon^{\alpha-1}= \pm\left[9 T^{2}(0)+6 T(2 \epsilon) T(0)+T^{2}(2 \epsilon)-16 T^{2}(\epsilon)\right]^{1 / 2}$, where $Q=2 \pi \hbar^{\alpha} D_{\alpha} /\left(a^{\alpha-1} g\right)$. The energy equations (i) and (ii) are plotted in the left and right panels of Fig. 2, respectively, for $Q=2$. 


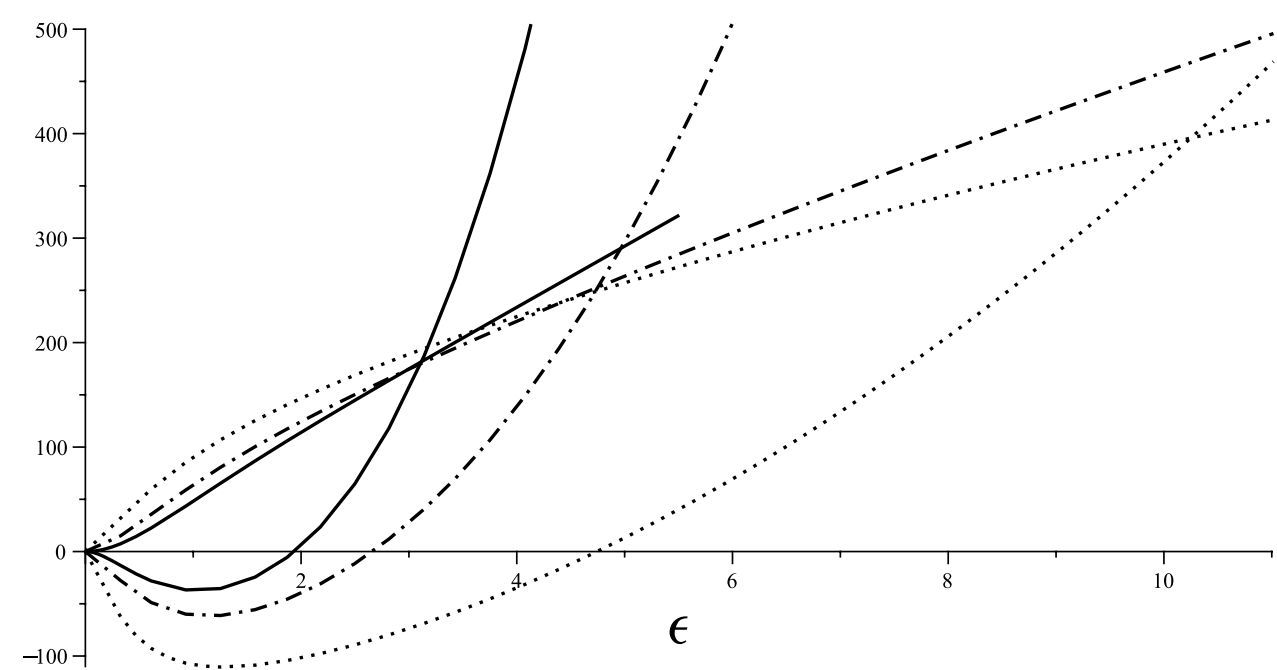

Fig. 1. Plots of the functions $f(\alpha, \epsilon)$ and $h(\alpha, \epsilon)$ for the case $V_{0}>0$ with $R=2$ and $\alpha=1.6$ (dotted curve), $\alpha=1.8$ (dash-dotted curve), and $\alpha=2.0$ (solid curve).
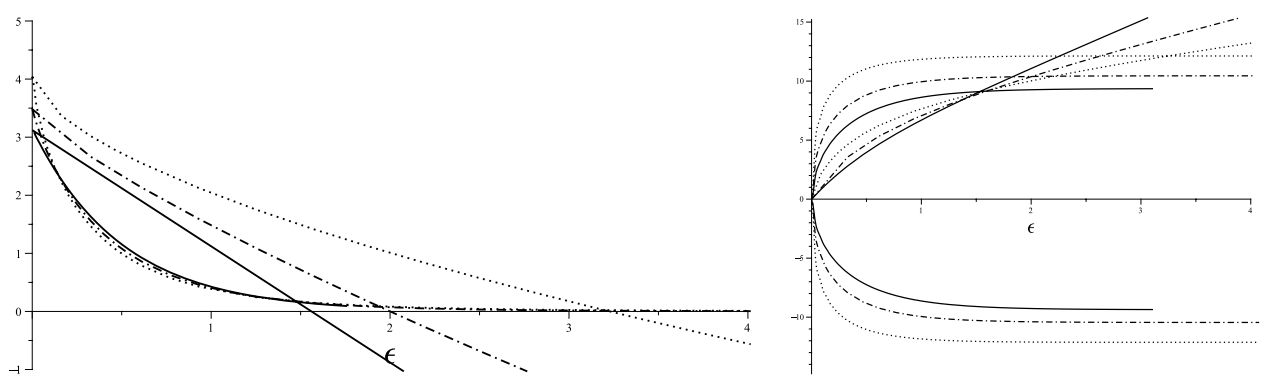

Fig. 2. Plots of the energy equations for the case $V_{0}<0$ with $Q=2$ and $\alpha=1.6$ (dotted curves), $\alpha=1.8$ (dash-dotted curves), and $\alpha=2.0$ (solid curves).

The wave function $\psi(x)$ can be obtained by inverse Fourier transforming $\tilde{\psi}(p)$. Furthermore, by a suitable choice of phase and application of Parseval's theorem, ${ }^{3}$ $\int_{-\infty}^{\infty} \psi^{*}(x) \psi(x) d x=(2 \pi \hbar)^{-1} \int_{-\infty}^{\infty} \tilde{\psi}^{*}(p) \tilde{\psi}(p) d p$, the normalized wave function for the case $V_{0}>0$ can be expressed as $\Psi(x)=N_{\alpha}[W \phi(x+a)-\phi(x)+Z \phi(x-a)]$, where $N_{\alpha}=\sqrt{\pi \lambda / \hbar} F(\alpha, W, Z)$ is the normalization constant, with $F(\alpha, W, Z)=\left[\alpha^{-2}(\alpha-\right.$ 1) $\left.\pi\left(W^{2}+Z^{2}+1\right) \csc (\pi / \alpha)-4(W+Z) I(a \lambda / \hbar)+2 W Z I(2 a \lambda / \hbar)\right]^{-1 / 2}$ from which we define $W=C_{0}(a) / 2 C_{1}(0), Z=C_{2}(a) / 2 C_{1}(0)$, and $I(y)=\int_{0}^{\infty} \cos (y q)\left(q^{\alpha}+1\right)^{-2} d q$ $(q=p / \lambda)$, and the $\phi$ 's are expressed in terms of Fox's $H$-function: ${ }^{5}$

$$
\phi(y)=H_{2,3}^{2,1}\left[\left.(\lambda / \hbar)^{\alpha}|y|^{\alpha}\right|_{(0, \alpha),(1-1 / \alpha, 1),(1 / 2, \alpha / 2)} ^{(1-1 / \alpha, 1),(1 / 2, \alpha / 2)}\right] .
$$

Figure 3 plots this bound-state wave function for $W=Z$. 


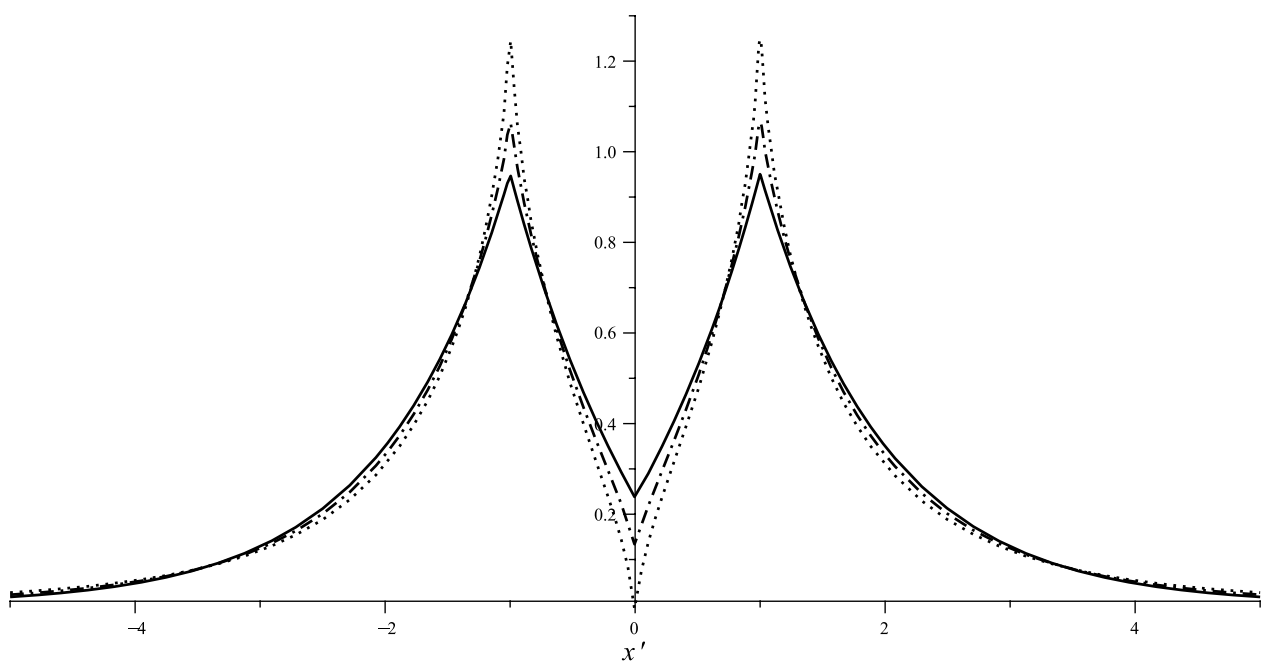

Fig. 3. Plot of the wave function $\Psi / N_{\alpha}$ as a function of $x^{\prime}=x \lambda / \hbar$ for $\alpha=1.6$ (dotted curve), $\alpha=1.8$ (dash-dotted curve), and $\alpha=2.0$ (solid curve) with $W=Z=2$.

Case II: $E>0$. For this case we let $\lambda^{\alpha}=E / D_{\alpha}(\lambda>0)$. Using the property $f(x) \delta(x)=f(0) \delta(x)$ of the delta function (see also Ref. 3 for the treatment of single and double delta potentials for the case $E>0$ using this property), we write $\tilde{\psi}(p)$ as $\tilde{\psi}(p)=A_{1} \delta(p-\lambda)+A_{2} \delta(p+\lambda)-\gamma_{\alpha}\left(|p|^{\alpha}-\lambda^{\alpha}\right)^{-1}\left[e^{i a p / \hbar} C_{0}(a)-2 C_{1}(0)+e^{-i a p / \hbar} C_{2}(a)\right]$. Using this to find $C_{0}, C_{1}, C_{2}$ we obtain

$$
\begin{aligned}
& U C_{0}(a)=\frac{\mu\left(2 \rho l_{1}-3 l_{2}^{2}+1\right)}{1-l_{2}} M_{0}(a \lambda / \hbar)+2 \mu \rho M_{1}(0)+\frac{2 \mu\left(l_{2}-\rho l_{1}\right)}{1-l_{2}} M_{2}(a \lambda / \hbar), \\
& U C_{1}(0)=-\mu \rho M_{0}(a \lambda / \hbar)+\nu\left(1+l_{2}\right) M_{1}(0)-\mu \rho M_{2}(a \lambda / \hbar), \\
& U C_{2}(a)=\frac{2 \mu\left(l_{2}-\rho l_{1}\right)}{1-l_{2}} M_{0}(a \lambda / \hbar)+\mu \rho M_{1}(0)+\frac{2 \mu\left(2 \rho l_{1}+1\right)}{1-l_{2}} M_{2}(a \lambda / \hbar),
\end{aligned}
$$

where we denote $\mu=\left[1+\Lambda_{\alpha}^{-1} S(0)\right]^{-1}, \nu=\left[1-2 \Lambda_{\alpha}^{-1} S(0)\right]^{-1}, \rho=\Lambda_{\alpha}^{-1} \nu S(a \lambda / \hbar)$, $l_{j}=\Lambda_{\alpha}^{-1} \mu S(j a \lambda / \hbar)(j=1,2), U=4 \rho l_{1}+l_{2}+1, M_{0}(a \lambda / \hbar)=M_{2}(-a \lambda / \hbar)=$ $A_{1} e^{-i a \lambda / \hbar}+A_{2} e^{i a \lambda / \hbar}, M_{1}(0)=A_{1}+A_{2}$, and $S(y)=2 \int_{0}^{\infty} \cos (y q)\left(q^{\alpha}-1\right)^{-1} d q$ $(q=p / \lambda)$; note from these definitions that $\nu l_{1}=\mu \rho$.

The wave function $\psi(x)$ after inverse Fourier transforming $\tilde{\psi}(p)$ is $\psi(x)=$ $A_{1}^{\prime} e^{i \lambda x / \hbar}+A_{2}^{\prime} e^{-i \lambda x / \hbar}+\left(2 \hbar \Lambda_{\alpha}\right)^{-1}\left[C_{0}(a) \zeta(x+a)-2 C_{1}(0) \zeta(x)+C_{2}(a) \zeta(x-a)\right]$, where $A_{j}^{\prime}=A_{j} / 2 \pi \hbar(j=1,2)$, the $C_{n}$ 's are those enumerated in Eqs. (6)-(8), and the $\zeta$ 's 
are the following Fox's $H$-functions:

$$
\begin{aligned}
\zeta(y)= & H_{2,3}^{2,1}\left[\left.(\lambda / \hbar)^{\alpha}|y|^{\alpha}\right|_{(0, \alpha),(1-1 / \alpha, 1),(1-[2+\alpha] / 2 \alpha,[2+\alpha] / 2)} ^{(1-1 / \alpha, 1),(1-[2+\alpha] / 2 \alpha,[2+\alpha] / 2)}\right] \\
& -H_{2,3}^{2,1}\left[\left.(\lambda / \hbar)^{\alpha}|y|^{\alpha}\right|_{(0, \alpha),(1-1 / \alpha, 1),(1-[2-\alpha] / 2 \alpha,[2-\alpha] / 2)} ^{(1-1 / \alpha, 1),(1-[2-\alpha] / 2 \alpha,[2-\alpha] / 2)}\right] .
\end{aligned}
$$

\section{Acknowledgments}

We are grateful to Dr. C. Bernido and Dr. M. V. Carpio-Bernido for their hospitality during our stay in Jagna for the workshop.

\section{References}

1. N. Laskin, Fractional quantum mechanics, Phys. Rev. E 63, 3135 (2000).

2. N. Laskin, Fractional Schrödinger equation, Phys. Rev. E 66, 056108 (2002).

3. E. C. de Oliveira, F. S. Costa, and J. Vaz, Jr., The fractional Schrödinger equation for delta potentials, J. Math. Phys. 51, 123517 (2010).

4. S. H. Patil, Quadrupolar, triple $\delta$-function potential in one dimension, Eur. J. Phys. 30, 629 (2009).

5. A. M. Mathai, R. K. Saxena, and H. J. Haubold, The H-Function: Theory and Applications (Springer, New York, 2009). 